Original article

\title{
The value of Tuffier's line for neonatal neuraxial procedures
}

Albert van Schoor ${ }^{1}$, MC Bosman ${ }^{1}$, and AT Bosenberg ${ }^{2}$,

${ }^{1}$ Department of Anatomy, School of Medicine, Faculty of Health Sciences, University of Pretoria, Pretoria, South Africa.

${ }^{2}$ Department of Anesthesiology and Pain Management, University Washington and Seattle Children's Hospital, Seattle, USA.

Numbers of tables: 2

Numbers of figures: 3

Abbreviated title: Tuffier's line in neonates

${ }^{*}$ Correspondence to:

Dr. A. van Schoor,

Department of Anatomy, Section of Clinical Anatomy, School of Medicine, Faculty of Health Sciences, University of Pretoria, Private Bag X323, Arcadia, Pretoria, 0007, South Africa

Tel: +27 123192315

Telefax: +27 123192240

E-mail: albert.vanschoor@up.ac.za 


\section{ABSTRACT}

The spine of L4 usually lies on a line drawn between the highest points of the iliac crests (Tuffier's line) in adults. Although its accuracy has been questioned, it is still commonly used to identify the spinous process of the $4^{\text {th }}$ lumbar vertebra before performing lumbar neuraxial procedures. In children, this line is said to cross the midline at the level of L5. A literature search revealed that the description this surface anatomical line is vague in neonates. The aims of this study were to determine the vertebral level of Tuffier's line, as well as its distance from the apex of the sacrococcygeal membrane (ASM), in 39 neonatal cadavers in both a prone and flexed position. It was found that when flexed, Tuffier's line shifted from the level of L4/L5 (prone position) to the upper third of L5. The mean distance from the ASM to Tuffier's line was $23.64 \mathrm{~mm}$ when prone and $25.47 \mathrm{~mm}$ when flexed, constituting a statistically significant increase in the distance $(p=0.0061)$. Therefore, in the absence of advanced imaging modalities, Tuffier's line provides practitioners with a simple method of determining a level caudal to the termination of the spinal cord, at approximately the L4/L5 in a prone neonate and the upper margins of L5 when flexed.

Key words: Inter-cristal line, lumbar spinous process, lumbar puncture, spinal anesthesia, lumbar epidural, pediatric, child 


\section{INTRODUCTION}

Several rat, other rodents and some primate studies have recently suggested that general anesthesia is neurotoxic i.e. there is an increase in programmed cell death (apoptosis) in the brain during a crucial stage of neonatal development after inhalational anesthesia or ketamine (Wang \& Slikker, 2008; Perouansky \& Hemmings, 2009; Sanders \& Davidson, 2009). Although there is still some controversy, it is only logical that the possible deleterious effects of inhalational anesthesia could be minimized by reducing the exposure to general anesthetic agents by using it in combination with regional anesthesia. In these cases, a much lower dose of general anesthesia is administered, which acts as a sedative to keep the child asleep and unaware while the local anesthetic agent is administered (Bosenberg, 2012). This combination has become standard pediatric anesthetic practice and has been proven to be a safe and effective method of analgesia in a pediatric population (Barrington \& Scott, 2008; Bosenberg, 2012).

The effective use of any neuraxial procedures in neonates, however, depends on precise knowledge of the segmental arrangement of the motor and sensory nerves, an appreciation of the increasing obliquity of the nerve roots and knowledge of the surface anatomy that relates to the vertebral canal. Only when all of the above elements are understood can the precise vertebral level for the performance of lumbar epidural blocks, spinal anesthesia, and lumbar puncture 
be appropriately selected. In the thoracic region, the spinous processes of the vertebrae are easily palpable; the spine of $T 7$, which overlies the level of the body of T8 (and is at the level of the spinal cord segment of T9/T10) lies at the level of the inferior angle of the scapula when in the anatomical position. By counting the vertebral spines from this point, one can identify the spines of the other thoracic vertebrae (Craven, 2004, Snider et al., 2011).

Regardless of its accuracy, Tuffier's (or the inter-cristal) line is the most common method of identifying the correct lumbar vertebral levels in adults (Reynolds, 2000; Chakraverty et al., 2007; Windisch et al., 2009; Snider et al., 2011). This line is drawn between the superior aspects of the iliac crests. The belief is that this line will transect the spinous process of L4 - L5 (Kim et al., 2003).

Although research regarding Tuffier's line in children is vague, studies have reported that Tuffier's line crosses the vertebral column approximately at the level of L5/S1 in children (Jankovic and Wells, 2001; Tame and Burstal, 2003). From this point, other vertebral levels can be identified.

The accuracy of Tuffier's line has been called into question many times in scientific publications. It would also appear that there will be a high degree of inaccuracy when using a single anatomical landmark, such as the iliac crests or Tuffier's line, to identify correct needle placement (Lirk et al., 2004; Snider et al., 
2011). It has also been suggested that Tuffier's line is significantly more caudal in women, transecting the spinous process of L5, instead of L4 in men. As Snider and co-workers concluded in their study; it is more accurate to use multiple bony landmarks to identify the lumbar spinous processes than when only single anatomical landmarks are used. They suggested identifying (a) T12 by the smaller size of its spinous process compared with that of L1 to determine the location of $\mathrm{L} 1$; (b) the $12^{\text {th }}$ ribs and their attachment site at T12 to determine the location of T12 and its spinous process and subsequently the location of L1; (c) Tuffier's line to locate the spinous process of $L 4$; (d) the sacral base to determine the location of L5; and (e) the L5 spinous process by the smaller size of its spinous process to determine the location of L4 (Snider et al., 2011). Health professionals, mostly in developed countries, have the added benefit of being able to use advanced imaging modalities such as ultrasonography, fluoroscopy or computed tomography to correctly identify the lumbar spinous processes, and therefore the correct needle placement site (Wagner, 2004; Ferre \& Sweeney, 2007).

Many health care institutions in the developing world cannot provide or do not have costly imaging equipment that could be used to accurately identify the spinous processes during lumbar neuraxial procedures. In these cases, the anatomical and practical knowledge of the practitioner performing the procedure is essential to correctly identify the needle placement site(s). Another limitation of using multiple anatomical landmarks in neonatal patients, as suggested by 
Snider et al. (2011), is that the spinous processes of the lumbar vertebrae are still developing and difficult to palpate. This includes the $12^{\text {th }}$ rib and its articulation to T12. This makes Tuffier's line the most practical means of identifying the lumbar vertebral level.

The aim of this study was therefore to determine the vertebral level, in both a prone and a flexed position, where Tuffier's line transects the vertebral column of a neonatal cadaver sample. The second aim was to determine if the vertebral level of Tuffier's line changes with positioning i.e. when a prone neonatal cadaver is flexed.

\section{MATERIALS AND METHODS}

A sample of 39 neonatal cadavers (mean length: $0.42 \mathrm{~m} \pm 0.07 \mathrm{~m}$; mean weight: $1.59 \mathrm{~kg} \pm 0.85 \mathrm{~kg}$ ) was used in this study. All cadavers were legally obtained (according to the South African National Health Act, number 61 of 2003) and stored in the Department of Anatomy, University of Pretoria, South Africa for research and teaching purposes. Ethical clearance to perform this study on the sample of neonatal cadavers was first obtained from the Main Ethics Committee of the Faculty of Health Sciences, University of Pretoria.

The lumbar and sacral regions of the neonatal cadavers were carefully dissected in order to expose the laminae and spinous processes of the lumbar 
vertebrae. During the dissection, the two iliac crests and the sacrococcygeal membrane, overlying the sacral hiatus, were also exposed. The most superior border of each iliac crest and the apex of the sacrococcygeal membrane (ASM) were then marked. High quality digital photographs were taken of the dissected vertebral column in both a prone and a flexed position (flexed between 40-50 degrees at the level of the umbilicus). Flexion of the neonatal cadavers aimed to simulate as accurately as possible that of a living neonate or infant. A photo scale (measured in $\mathrm{mm}$ ) was placed on top of the dissected area (without covering any of the relevant structures) before each photograph was taken.

On the photograph, each vertebra was divided into thirds. Each third, as well as each interlaminar space, was given a corresponding number, i.e., the upper third of T12 was "1", the middle third of T12 was " 2 ", the lower third of T12 was " 3 " and the T12/L1 intervertebral disc was " 4 ". Numbering then continued from the upper border of L1 (which was " 5 ") to the L5/S1 intervertebral disc (which was number "24"). A straight line was also drawn between the two iliac crests, which were marked on each digital photo. The vertebral level (indicated by a corresponding number) where this line intersected the vertebral column was then noted in both the prone and flexed position (see Figure 1). 


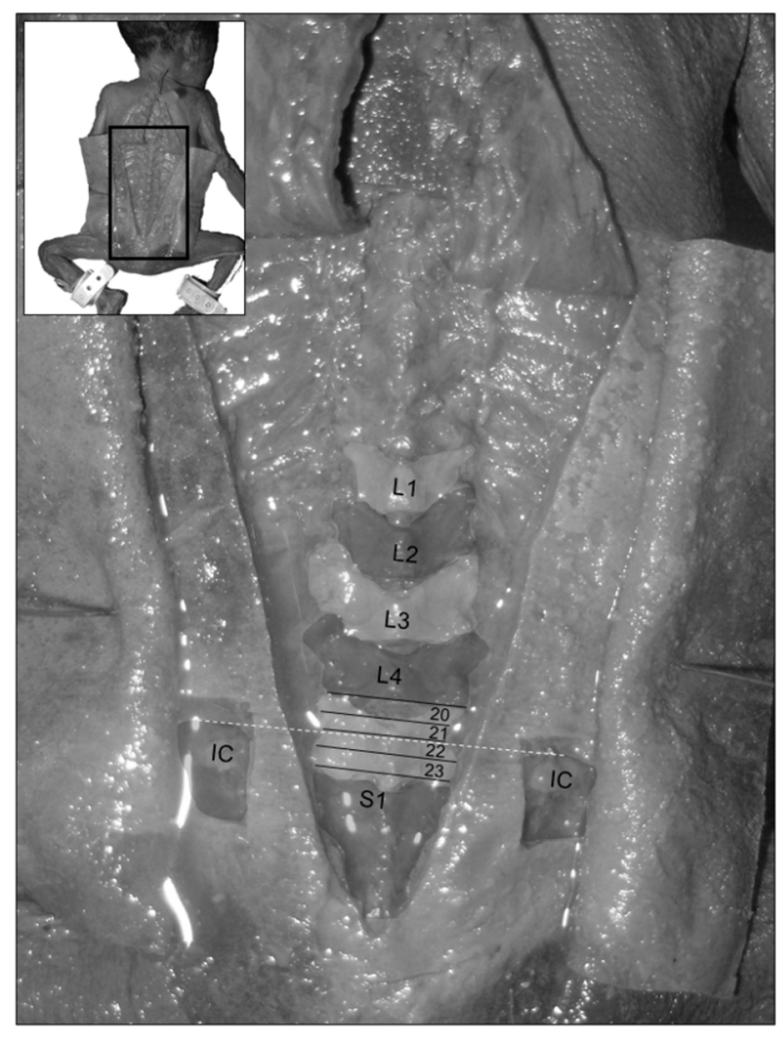

Figure 1: The exposed lumbar vertebrae of a neonatal cadaver in a prone position. The numerical division of the $5^{\text {th }}$ lumbar vertebra and Tuffier's line (dashed line between the two exposed iliac crests (IC)) are also indicated.

The photographs were analyzed by using UTHSCSA Image Tool V3.0. Using the Calibrate Spatial Measurements function, the photo scale (found on each photograph) was converted into a pixel format. This allowed for accurate measurements of the photographs by means of the Distance function, which converts the length of a straight line drawn between two points on the photograph into millimeters. The distance of the ASM to where Tuffier's line crosses the vertebral column in both a prone and flexed position was measured. These measurements were recorded on a MS Excel ${ }^{\mathrm{TM}}$ worksheet and statistical analysis of the data was performed. The actual and percentage change in the 
distance of the ASM to Tuffier's line was also determined for each neonate in both positions.

\section{RESULTS}

The corresponding number of the vertebral level where Tuffier's line intersects the lumbar vertebral column is summarized in Table 1.

Table 1: Mean vertebral level, in both a prone and flexed position, of Tuffier's line in a neonatal sample. The corresponding number of each division is given in brackets.

\begin{tabular}{c|cc} 
& \multicolumn{2}{|c}{ Vertebral level of Tuffier's line } \\
\cline { 2 - 3 } & Prone & Flexed \\
\hline Mean & L4/L5 (20) & Upper third L5 (21) \\
Lower range* & $1.87^{*}$ & $1.81^{*}$ \\
Upper range* & Lower third L4 (19) & L4/L5 (20) \\
*Ranges are all with a 95\% confidence interval &
\end{tabular}

It is clear from this sample that, when the neonate is in a prone (or neutral) position, Tuffier's line crosses the vertebral column at the L4/L5 interlaminar space (95\% confidence level, range between the lower third of L4 and the L4/L5 interlaminar space). During flexion this level moves caudally by an average of 
one number position (0.97) to the upper third of the L5 vertebra (95\% confidence interval, range between the L4/L5 interlaminar space and the upper third of L5).

The distance from the ASM to Tuffier's line, with the neonate in both a prone and flexed position, was also measured using Image Tool and the results are summarized in Table 2.

Table 2: Measurement of the ASM to Tuffier's line, in both a prone and flexed position.

\begin{tabular}{c|cc|c} 
& \multicolumn{2}{|c|}{ Distance in $\mathrm{mm}$} & $\begin{array}{c}\% \text { Change } \\
\text { from prone }\end{array}$ \\
\hline Mean & Prone & Flexed & to flexed \\
SD & 5.64 & 25.47 & - \\
Lower range* $^{*}$ & 21.86 & 23.71 & - \\
Upper range* $^{*}$ & 25.41 & 27.24 & $11.10 \%$
\end{tabular}

${ }^{*}$ Ranges are all with a 95\% confidence interval

While the vertebral level of Tuffier's line moves caudally during flexion, the distance from the ASM to Tuffier's line increases significantly $(p=0.0061)$ from $23.64 \mathrm{~mm} \pm 5.65 \mathrm{~mm}$ (mean $\pm \mathrm{SD})(95 \%$ confidence interval; range: $21.86 \mathrm{~mm}-$ $25.41 \mathrm{~mm})$ to $25.47 \mathrm{~mm} \pm 5.63 \mathrm{~mm}(95 \%$ confidence interval; range $23.71 \mathrm{~mm}-$ $27.25 \mathrm{~mm})$. This constitutes a percentage change that ranges from $3.10 \%$ to 
$11.10 \%$ (95\% confidence interval) in the distance between ASM and Tuffier's line.

\section{DISCUSSION}

Tuffier's line is commonly used to determine the L4 vertebral level when performing procedures such as lumbar epidural blocks, spinal anesthesia, or lumbar punctures in adults (Reynolds, 2000). This is despite Reynolds (2001) describing seven cases where neurological damage followed spinal or combined spinal-epidural anesthesia in adult women. He therefore believes that Tuffier's line is unreliable in determining the correct lumbar vertebral level. Because of this, Reynolds as well as Boon and colleagues (2004) recommend rather selecting one space lower than indicated by Tuffier's line, as the identified space is likely to be at least one interlaminar space too high.

Generally, the line is described as crossing the vertebral column at the level of the L4/L5 interlaminar space (Quinnell and Stockdale, 1983), or the L4 spinous process (Render, 1996). These studies were all performed without lumbar flexion. However, patients are normally flexed to increase the area of the lumbar interlaminar spaces and they are placed either on their side (lateral decubitus position) or sitting up when diagnostic spinal punctures or lumbar spinal or epidural blocks are performed. Because of this, Kim and co-workers (2003) questioned the validity of these initial findings regarding Tuffier's line. 
They conducted a study where they examined 103 lumbar spine X-rays of patients in both a neutral and flexed position. They found that Tuffier's line was in line with the L4/L5 interlaminar space in a neutral position, as well as in a fully flexed position. In relation to the vertebral column, when flexed, Tuffier's line moved cranially in $1.9 \%$, it moved caudally in $15.5 \%$ and remained on the same level in $82.5 \%$ of their sample.

Pediatric anesthesia textbooks contend that Tuffier's line crosses the midline in the area of about L5 in infants and at about L5/S1 in neonates (Jankovic and Wells, 2001). Many studies in the past are based on X-ray findings which can be misleading since the cartilaginous portion of the iliac crest is not radio-opaque and therefore not seen on X-ray images. Tame and Burstal (2003) evaluated the vertebral level of Tuffier's line in MRIs of 35 children less than ten years old. They found that the line intersected the L5 vertebra (with an interquartile range of 0.5 vertebral levels). These MRIs were evaluated with the children in a neutral position.

The results obtained from the neonatal sample, in a prone/neutral position, concur with what Kim et al. (2003) found in their study. Tuffier's line intersects the L4/L5 level (95\% confidence interval, range: lower third of L4 to L4/L5) in $25.64 \%$ of the sample (see Figure 2), this is followed by the middle third of L4 $(20.51 \%)$ and then the upper and middle thirds of L5 (both 17.95\%). With flexion, Tuffier's line moved caudally to the upper third of L5 (95\% confidence interval, range: 
L4/L5 to upper third of L5), with the upper and middle thirds of L5 being the most common level where Tuffier's line transects the vertebral column $(20.51 \%$ respectively).

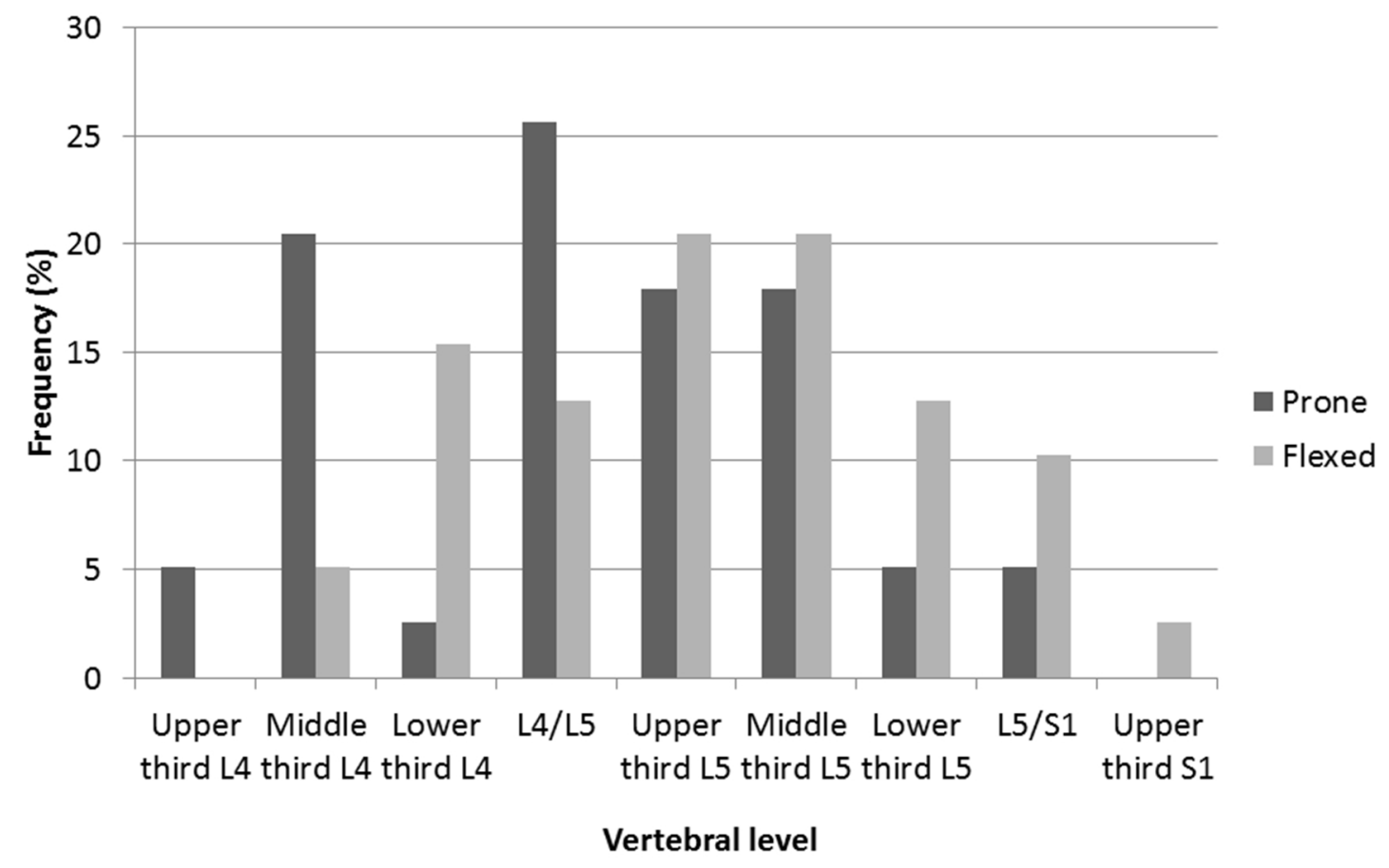

Figure 2: Frequency of the level of Tuffier's line in a neonatal cadaver population.

However, in the cadaver sample, only nine $(23.08 \%)$ of the sample had no change in number position when flexed, whereas the level moved caudally by one number position in 22 neonates $(56.41 \%)$ and two levels in eight neonates (20.51\%). In this study, a level is represented by a third of a vertebral body or the length of an intervertebral disc and not an entire vertebral body. It was clear that flexion of the neonatal cadaver effectively "stretches" the vertebral column, thereby increasing the distances between the lamina of adjacent vertebrae (in 
particular the lumbar vertebrae). The ilia, attached to the sacrum, remain in a more constant position. The theory is therefore that the relationship between the iliac crests (and therefore Tuffier's line) and the vertebral level changes as the neonate is flexed. Since the iliac crests stay in a more constant position, Tuffier's line will be in a slightly more caudal position as the results indicated. These changes are small (one to two thirds of a vertebra), but clinically relevant.

In contrast to other studies, Tuffier's line crosses the vertebral column, in the neutral or prone position, at the level of the L4/L5 interlaminar space (see Figure 3), and this level tends to move caudally when the neonate is flexed.

There are several reasons that could explain this. In the cadaver sample, the iliac crests were completely exposed through dissection, and the superior margin of the iliac crests included the cartilaginous rim of the iliac crests. Normally the iliac crests are still cartilaginous throughout early life, and even into adolescence (Scheuer and Black, 2000). Errors in identifying the correct vertebral level could therefore be made if these anatomical landmarks are not identified correctly. This will result in the vertebral level to be incorrectly identified at a level more caudal, as seen in the study by Tame and Burstal (2003). The study conducted by Kim et al. (2003) showed that in adults, the level of Tuffier's line did not change in the majority of their sample. In this study, each vertebra was subdivided into three equal parts and changes in "level" could occur at the same vertebral level (i.e., from the upper third of $L 4$ to the lower third of L4). Therefore, 


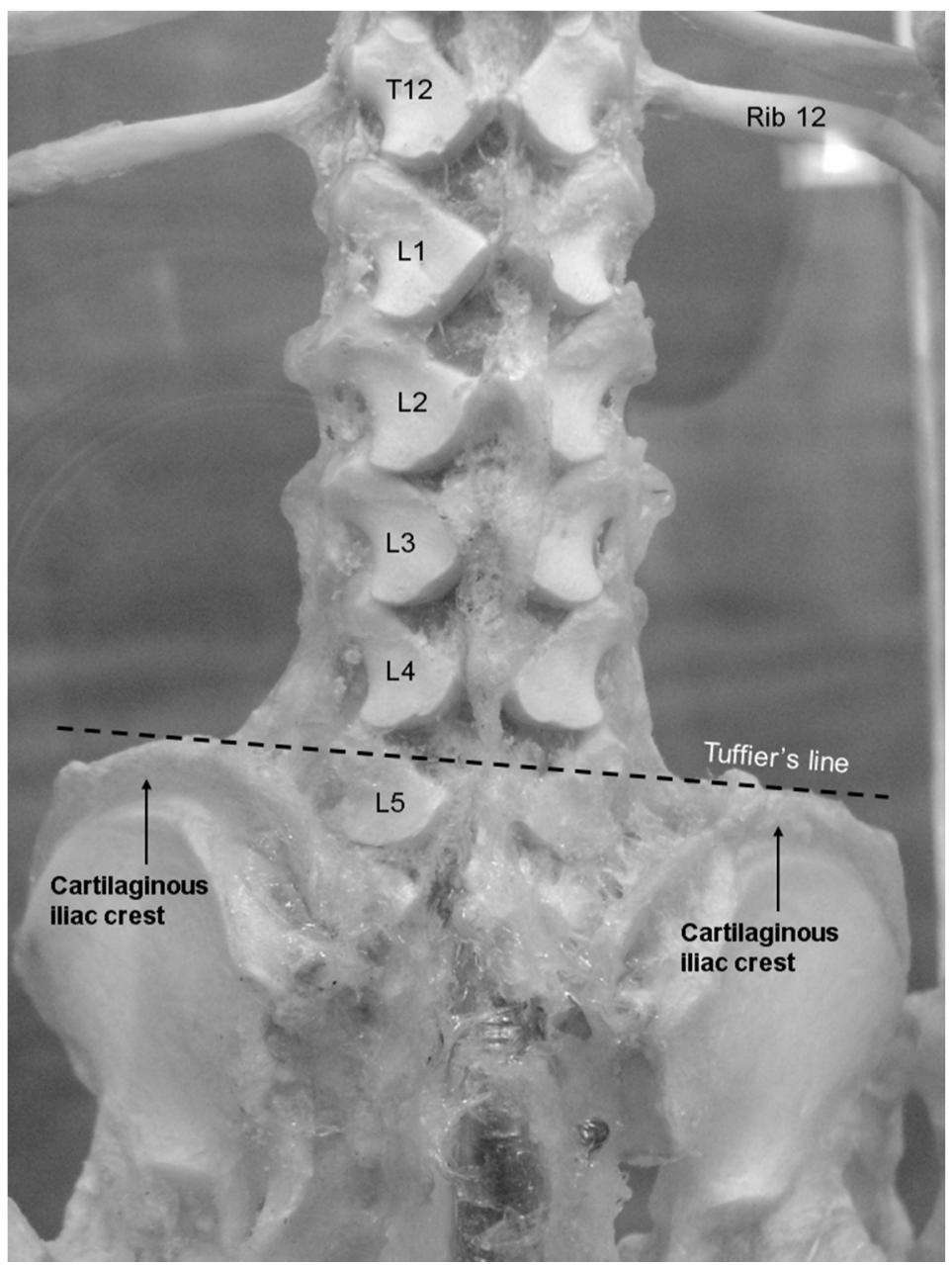

Figure 3: Posterior view of an articulated neonatal vertebral column and iliac crests. The $12^{\text {th }}$ thoracic and $5^{\text {th }}$ lumbar vertebrae are indicated, as well as Tuffier's line (black dashed line).

the results of this study would suggest that the level of Tuffier's line move caudally with flexion of the trunk. This is an important factor to bear in mind, during needle placement even though it is not a substantial change it can be clinically significant in neonatal or very young patients. In this sample, Tuffier's line was never found to be more cranial than the upper third of the L4 in the prone sample and the middle third of L4 when flexed. The most caudal level, in 
the prone position, was observed at the L5/S1 interlaminar space and, in the flexed position, at the upper third of S1.

Limitations in this study include the lack of specific information regarding the neonatal sample. Firstly it was impossible to determine whether the neonatal cadavers, which ranged from very low birth weight $(<1.5 \mathrm{~kg})$, low birth weight $(1.5 \mathrm{~kg}-2.5 \mathrm{~kg})$ and normal birth weight $(>2.5 \mathrm{~kg})$, were growth retarded term or preterm infants. This could have confounded the results obtained; however this was likely to be minimized by the large sample size and by exclusion of neonates with abnormal morphology. Secondly, even though flexion of the neonatal cadavers was done consistently and aimed to simulate the clinical situation , there is an obvious difference between embalmed cadaveric and living tissue. However, for the purpose of observing the changes that occur during flexion and its implication in the clinical setting, these differences in tissue could be considered to be negligible.

Although one seldom encounters obese neonates, which would make the identification of Tuffier's line difficult, accurate identification of the superior margins of the iliac crests is still very important. Practitioners aiming to use Tuffier's line should remember that the iliac crests have yet to ossify completely and still have a cartilaginous rim, which is not visible on a radiograph. This could be misleading as Tuffier's line may be seen on X-ray to be more caudad than expected. Despite this, in the absence of advanced imaging modalities, Tuffier's 
line provides practitioners with a simple means of determining a level below the termination of the spinal cord, at approximately the L4/L5 in a prone neonate and the upper margins of $L 5$ when the neonate is flexed.

\section{CONFLICT OF INTEREST}

There is no conflict of interest to declare by any of the authors.

\section{REFERENCES}

Barrington MJ, Scott DA. 2008. Do we need to justify epidural analgesia beyond pain relief? Lancet 372:514-516.

Boon JM, Abrahams PH, Meiring JH, Welch T. 2004. Lumbar puncture for the generalist. SAFP 46:38-42.

Bosenberg AT. 2012. Benefits of regional anesthesia in children. Pediatric Anesth 22:10-18.

Chakraverty R, Pynsent P, Isaacs K. 2007. Which spinal levels are identified by palpation of the iliac crests and the posterior superior iliac spines? J Anat 210:232-236.

Craven J. 2004. Anatomy relevant to epidural and subarachnoid blockade. Anaesth Intensive Care Med 5(5):147-149. 
Ferre RM, Sweeney TW. 2007. Emergency physicians can easily obtain ultrasound images of anatomical landmarks relevant to lumbar puncture. Am J Emerg Med 25:291-296.

Jankovic D, Wells C. (eds.) 2001. Regional Nerve Blocks, 2nd Ed. Vienna: Blackwell Science Berlin.

Kim J, Jung C, Lee J, Min S, Bahk J. 2003. Influence of lumbar flexion on the position of the intercrestal line. Reg Anesth Pain Med 28:509-511.

Lirk P, Messner H, Deibl M, Mitterschiffthaler G, Colvin J,Steger B, et al. 2004. Accuracy in estimating the correct intervertebral space level during lumbar, thoracic and cervical epidural anaesthesia. Acta Anaesthesiol Scand 48:347-349.

Perouansky M, Hemmings HC. 2009. Neurotoxicity of general anesthetics. Anesthesiology 11: 365-1371.

Quinnell RC, Stockdale HR. 1983. The use of in vivo lumbar discography to assess the clinical significance of the position of the intercrestal line. Spine 8:305-307.

Render CA. 1996. The reproducibility of the iliac crest as a marker of lumbar spine level. Anaesthesia 51:1070-1071.

Reynolds F. 2000. Logic in the safe practice of spinal anesthesia (editorial). Anaesthesia 55:1045-1046.

Reynolds F. 2001. Damage to the conus medullaris following spinal anaesthesia. Anaesthesia 56(3):238-247. 
Sanders RD, Davidson A. 2009. Anesthetic induced neurotoxicity of the neonate: time for clinical guidelines? Pediatric Anesth 19:1141-1146.

Scheuer L, Black S. (eds.) 2000. Developmental Juvenile Osteology. San Diego: Academic Press.

Snider KT, Snider EJ, Degenhardt BF, Johnson JC, Kribs JW. 2011. Palpatory accuracy of the lumbar spinous processes using multiple bony landmarks. J Manipulative Physiol Ther 34:306-313.

Tame SJ, Burstal R. 2003. Investigation of the radiological relationship between iliac crests, conus medullaris and vertebral level in children. Paediatr Anaesth 13(8):676-680.

Wagner AL. 2004. CT fluoroscopy-guided epidural injections: technique and results. AJNR Am J Neuroradiol 25:1821-1823.

Wang C, Slikker W Jr. 2008. Strategies and experimental models for evaluating anesthetics: effects on the developing nervous system. Anesth Analg 106:1643-1658.

Windisch G, Ulz H, Feigl G. 2009. Reliability of Tuffier's line evaluated on cadaver specimens. Surg Radiol Anat 31:627-630. 